\title{
Índice de massa corporal (massa corporal.estatura-2) como indicador do estado nutricional de adultos: revisão da literatura *
}

\section{Body mass index as a tool in the nutritional assessment of adults: a review}

\section{Luiz A. Anjos **}

\begin{abstract}
ANJOS, L.A. Índice de massa corporal (massa corporal.estatura ${ }^{-2}$ ) como indicador do estado nutricional de adultos: revisão da literatura. Rev. Saúde públ., S.Paulo, 26: 431-6,1992. São discutidas as virtudes, limitações e possíveis aplicações do índice de massa corporal (IMC), também chamado de índice de Quételet, que é calculado pela divisão da massa corporal em quilogramas pelo quadrado da estatura em metros, como indicador do estado nutricional de adultos. Baseado numa revisão ampla da literatura, os limites de corte para a definição de sobrepeso (IMC $\geq 25$ ) e desnutrição energética crônica (IMC < 18,5) são apresentados e discutidos. Conclui-se que apesar de não representar a composição corporal de indivíduos, a facilidade de sua mensuração e a grande disponibilidade de dados de massa corporal e estatura parecem ser motivos suficientes para a utilização do IMC em estudos epidemiológicos, em associação (ou não) a outras medidas antropométricas, até que metodologias de campo que expressem a composição corporal sejam desenvolvidas para tais estudos.
\end{abstract}

Descritores: Estado nutricional. Indice de massa corporal. Obesidade. Desnutrição protéico-calórica.

\section{Introdução}

A avaliação do estado nutricional de adultos requer o conhecimento das reservas energéticas e da massa metabolicamente ativa dos indivíduos sendo avaliados, o que se obtém através da avaliação da composição corporal (CC). Existem vários métodos para a avaliação da $\mathrm{CC}$ e mesmo os mais simples necessitam de treinamento especializado ${ }^{24}$. Portanto, é necessário o desenvolvimento de um indicador que incorpore medidas simples e expresse de algum modo as reservas energéticas do indivíduo. A presente revisão descreve as possiveis aplicações do índice de massa corporal na avaliação do estado nutricional de adultos em estudos epidemiológicos.

\section{Razoamento para o IMC}

A avaliação do estado nutricional de adultos era tradicionalmente feita através do conceito de "peso

\footnotetext{
* Estudo realizado com o apoio financeiro do $\mathrm{CNPq}$ (Proc.301076/89.8).

* Centro de Estudo da Saúde do Trabalhador e Ecologia Humana - Escola Nacional de Saúde Pública - Fundação Oswaldo Cruz - Rio de Janeiro, RJ - Brasil.
}

Separatas/Reprints: L.A. Anjos - Rua Leopoldo Bulhões, 1480 - Manguinhos - 21041-210 - Rio de Janeiro, RJ - Brasil. ideal" obtido pela comparação da massa corporal em função da estatura com um padrão antropométrico, geralmente a partir de dados de companhias de seguro. Se a massa corporal estivesse acima de $20 \%$ do padrão, dizia-se que a pessoa era obesa. Existe consenso sobre a inadequação da utilização de padrão único universal para adultos, já que há grande variação da estatura média das populações adultas do mundo. Portanto, existe a necessidade do desenvolvimento de indicadores antropométri$\cos$ do estado nutricional que reflitam a composição corporal, que sejam simples de se obter e que não necessitem de padrão para comparação ${ }^{46}$. A busca para o desenvolvimento de tais indicadores tem sido baseada nos dados de massa corporal (MC) e estatura (EST). Teoricamente, o indicador não deveria se correlacionar com EST, mas com a MC e outras medidas de gordura corporal. Tal indicador deve ser independente da estatura, já que um indivíduo mais alto terá massa corporal maior, decorrente da maior massa magra (osso, músculo e outras) e não necessariamente da massa gorda. Análises de grandes bancos de dados foram realizadas na tentativa de se encontrar a melhor relação MC.(EST) ${ }^{-1}$ que preenchesse os requisitos mencionados. Esses indicadores são genericamente chamados de "índices de massa corporal" pois incorporam relaçóes entre a MC e EST, e.g., MC.(EST) ${ }^{-1}$, MC.(EST ${ }^{-2}$ ), MCP.(EST) ${ }^{-9}$ (onde p e $q$ receberiam valores dependendo da amostra), entre outros. 
Há duas décadas, Keys e col. ${ }^{21}$ (1972) sugeriram chamar a relação MC.(EST $\left.{ }^{-2}\right)$ de Îndice de Massa Corporal (IMC), com a massa corporal expressa em quilogramas e a estatura em metros. A partir daí esta relação ficou popular na avaliação nutricional de adultos e alguns passaram a chamá-la também de índice de $Q$ uételet ${ }^{36} \mathrm{em}$ homenagem a seu criador $^{13}$. Isto se deve ao fato do IMC ser aparentemente o de melhor correlação com MC (valores do coeficiente de correlação " $r$ " normalmente superiores a 0,80 ) e, principalmente, pela sua baixa correlação com EST (normalmente " $r$ " em torno de 0,10 ), fato documentado internacionalmente em amostras de populações adultas 1,5,21,22,28,34,44. Entretanto, é fundamental enfatizar que estes dois requisitos não são suficientes para se recomendar a sua utilização universal ${ }^{37}$. É importante que se correlacionem os valores de IMC com outras medidas independentes de composição corporal, quais sejam, a massa de gordura corporal (MGC) ou o percentual de gordural corporal (\% GC). Em estudos com amostras representativas da população americana e de outros países do Primeiro Mundo, já foi demonstrado que o IMC correlaciona-se altamente com a massa de gordura corporal ( $\mathrm{r}$ em torno de $0,90)^{37}$, com a dobra cutânea subscapular ( $\mathrm{r}$ em torno de 0,70$)^{27}$, com a dobra cutânea tricipital (r acima de 0,60$)^{8}$, e com o percentual de gordura corporal (r acima de 0,70$)^{33}$. O IMC também se correlaciona em níveis altos com a circunferência abdominal ( $\mathrm{r}$ em torno de 0,80 ) como demonstrado no estudo longitudinal de Framingham ${ }^{16}$.

\section{Limitações}

Garn e col. ${ }^{9}$ (1986) enumeraram três limitações para o uso do IMC: a correlação com a estatura (que apesar de baixa ainda é significativa), com a massa livre de gordura (principalmente nos homens) e com a proporcionalidade corporal (relação tamanho das pernas/tronco), o que, segundo os autores, poriam em risco a utilização do IMC como indicador de gordura corporal. Baseado nesses argumentos e acrescentando ainda a importante influência da distribuição de gordura corporal à saúde (o que não é expresso pelo IMC), $\mathrm{McLaren}^{27}$ (1987) sugeriu o total abandono do uso do IMC em estudos de obesidade. Garrow ${ }^{12}$ (1988) concordou com as limitaçōes do IMC, mas mesmo assim enfatizou que "seria absurdo sugerir o abandono do IMC em estudos epidemiológicos", devido principalmente à ausência de outro indicador que seja tão simples e conveniente e para o qual existem tantos bancos de dados disponíveis. $\mathrm{Na}$ verdade, mesmo sabendo-se o grau de adiposidade, ou seja, o percentual de gordura corporal, e daí a quantidade total de massa magra e massa gorda, pode-se não conseguir distinguir os indivíduos saudáveis dos portadores de desnutrição energético protéica ${ }^{42}$. Por exemplo, indivíduos altos com desnutrição energética podem ter valores de massa magra similares àqueles de indivíduos mais bem nutridos, mas com estatura menor, já que a quantidade de massa magra está correlacionada com a estatura ${ }^{7}$. Portanto, VanItallie e col. ${ }^{42}$ (1990) sugerem a separação do IMC em índice de massa de gordura corporal (IMGC) e índice de massa livre de gordura (IMLG) que seriam calculados a partir das frações de gordura corporal e massa magra, respectivamente, superando, portanto, a principal limitação relacionada ao IMC. Apesar da vantagem teórica deste modelo, a grande restrição fica por conta da necessidade da mensuração da composição corporal, cujos métodos ainda não foram utilizados em grande escala em populaçð̃es de países em desenvolvimento e, portanto, o que tem, como conseqüência, não se saber a validade da sua utilização nessas populaçðes. Os dados disponíveis atualmente sugerem que, para a maior parte dos métodos de avaliação da composição corporal, os parâmetros assumidos como verdadeiros para um grupo populacional podem não ser os mesmos para outros grupos ${ }^{3,24}$. Consequientemente, as equaçōes de predição desenvolvidas a partir desses métodos são específicas para os grupos populacionais usados na sua obtenção, como evidenciado em amostra da população brasileira, onde as equaçōes de predição da composição corporal a partir das medidas de dobras cutâneas, produzidas em outros países, mostraram-se inadequadas para a amostra brasileira ${ }^{14}$.

São poucos os dados de IMC em populações de países em desenvolvimento. Os dados disponíveis indicam que as intercorrelações de IMC com a massa corporal e a estatura são semelhantes às encontradas em países desenvolvidos ${ }^{1,44}$. Estudos de composição corporal em países em desenvolvimento são ainda mais raros. Portanto, estudos precisam ser realizados nesses países de forma a comparar o IMC com medidas da composição corporal, para que se conheça o real potencial de utilização do IMC (e de suas fraç⿸̃es, IMGC e IMLG) na avaliação nutricional. De qualquer modo, tirando-se os extremos da magreza e excesso de corpulência, observados em alguns seguimentos da população (atletas e/ou trabalhadores que desenvolvem grande massa muscular), o IMC parece válido como indicador do estado nutricional em grupos de indivíduos, mas pode não sê-lo para indivíduos específicos ${ }^{41}$.

\section{Limites de Corte para Sobrepeso}

Não existe ainda uma definição clara dos limites de corte do IMC para definição do estado 
nutricional. Na verdade, existiu até certo impasse, já que uma comissão de especialistas reunidos pela Organização Mundial de Saúde ${ }^{45}$ sugeriu a adoção universal dos limites de corte norteamericanos, apesar do reconhecimento da falta de dados e, portanto, da validação desses cortes em populações de outras partes do mundo. Já outras comissőes de especialistas ${ }^{31,33}$ têm proposto a utilização dos tradicionais limites de corte sugeridos por vários autores ${ }^{2,11}$ que são: baixo peso (BP; IMC $<20)$, normal $(20 \leq$ IMC $<25$ ), sobrepeso (SP; $25 \leq$ IMC $<30$ ) e obeso (IMC $\geq 30$ ). Há, ainda, a proposta da separação de obesidade em graus (Garrow) ${ }^{10}$ : obesidade grau I seria quando o IMC ficasse entre 25 e 29,9 ; grau II quando o IMC ficasse entre 30 e 39,9 ; e grau III quando o IMC fosse superior a 40 . A utilização desta terminologia deve ser feita com cautela, já que, por definição, obesidade significa excesso de gordura corporal $^{4}$, o que, na verdade, não é medido através do $\mathrm{IMC}^{2,10}$. Mais recentemente um grupo de estudiosos da OMS acabou com a controvérsia ao sugerir o valor de 25 como limite máximo para normalidade ${ }^{47}$.

Outra metodologia sugerida para a definição de limites de corte é a determinação dos valores do IMC correspondentes aos percentis 85 e 95 de uma faixa etária, para diagnosticar sobrepeso e obesidade, respectivamente ${ }^{23}$. Com esta metodologia, estabelecem-se limites de corte específicos para a população em estudo, valores que poderão não servir para outros grupos populacionais, já que esses dados devem ser associados às características de saúde da população.

Problema associado a qualquer metodologia de determinação dos limites de corte é o fato de não se saber, claramente, como levar em consideração a idade, já que com o envelhecimento ocorre simultaneamente aumento na deposição de gordura e uma perda do tecido livre de gordura ${ }^{7}$. Em estudo longitudinal de 4 anos, Rookus e col. ${ }^{39}$ (1987) documentaram o aumento mediano do IMC, em homens e mulheres entre 19 e 35 anos de idade, de até 2,3 unidades de IMC. Infelizmente, os autores não fizeram avaliação da composição corporal e, portanto, não se consegue saber se houve, e se houve qual foi, a modificação da composição corporal nesses indivíduos. Deurenberg e col. ${ }^{6}$ (1989) estudaram a composição corporal de 72 idosos (idade entre 60 e 83 anos), aparentemente saudáveis ( 37 mulheres e 35 homens), e relacionaram os dados com o IMC. Apesar do IMC médio ficar dentro de níveis aceitáveis (entre 25 e $26 \mathrm{~kg}$. $\left(\mathrm{m}^{-2}\right)$, o percentual de gordura corporal foi alto ( $44 \%$ para as mulheres e $31 \%$ para os homens). Os autores sugerem que os valores normalmente utilizados de IMC devam ser adaptados para os idosos, já que o IMC indica um percentual de gordura corporal muito maior para os idosos do que para os indivíduos mais jovens. Bray ${ }^{2}$ (1992) sugeriu limites de corte na definição de sobrepeso, dependendo da idade. A tendência foi acrescentar uma unidade de IMC para cada década de vida a partir de 25 anos. Em amostra representativa da população francesa, Rolland-Cachera e col. ${ }^{38}$ (1991) observaram que os valores médios do IMC aumentaram aproximadamente de uma unidade de IMC para cada década de idade em ambos os sexos, com a tendência a cair após os 80 anos de idade. Recentemente, Must e col. ${ }^{29,30}$ (1991) produziram tabelas de distribuição do IMC em função da faixa etária, sexo e raça da população americana a partir dos dados da NHANES I. Esses autores sugerem a utilização dos valores de centis 85 e 95 do IMC para definição de obesidade e superobesidade, respectivamente. Os valores do centil 85 do IMC variaram de 26,14 a 31,58 , para as mulheres, e de 26,87 a 28,99 , para os homens de 20 anos a 70 anos de idade, respectivamente (Figura 1). Esses dados demonstram claramente aumento do IMC com o envelhecimento, o que representa aumento de gordura corporal. Existe, portanto, a preocupação e a necessidade de adequação dos limites de corte do IMC em função da faixa etária e sexo, que reflitam os menores riscos para a saúde. Entretanto, ainda não existe, na atualidade, consenso sobre como isso deva ser feito.

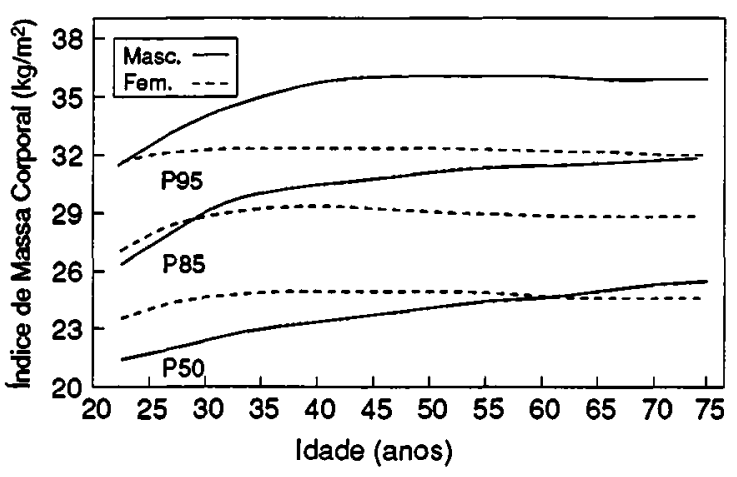

Figura 1. Medianas (P50), P85 e P95 do índice de massa corporal (IMC) da populaçăo americana em função do sexo e idade. Desenhado a partir de dados publicados da NHANES $129,30$.

\section{Limites de Corte para Desnutriçâo Energética Crônica}

Em países em desenvolvimento, além do diagnóstico de excesso de reserva energética (obesidade), permanece o desafio do diagnóstico da deficiência energética crônica em adultos. Ao contrário do que ocorre em crianças, não é aconselhável a utilização de dados de massa corporal e 
de estatura obtidos em populaçōes do Primeiro Mundo como referência, devido à grande variação da estatura do adulto em várias partes do mundo $^{26,46}$. Dados recentes da Pesquisa Nacional de Saúde e Nutrição (PNSN) de adultos brasileiros ${ }^{18}$ corroboram esta afirmação, visto que mesmo para o segmento da população de melhores condiçð̃es de renda, a mediana da estatura dos adultos brasileiros não alcança a estatura de populaçőes do Primeiro Mundo.

James e col. ${ }^{20}$ (1988) propuseram a utilização do IMC como indicador de deficiência energética crônica (DEC) em países do Terceiro Mundo. Valores de IMC inferiores a 16 indicariam DEC de terceiro grau e valores superiores a 18,5 indicariam normalidade. Os valores de IMC entre $16 \mathrm{e}$ 18,4 poderiam indicar os graus 1 e 2 de DEC, dependendo do nível de atividade física (NAF) ${ }^{19,45}$, medido através do gasto energético em relação à taxa metabólica basal. Estes limites foram recentemente sugeridos para serem utilizados em paises em desenvolvimento por um grupo de estudiosos da $\mathrm{OMS}^{47}$. Henry ${ }^{15}$ (1990) analisou os dados disponíveis na literatura sobre a relação entre o IMC e patologias que levam à morte e confirmou o valor de IMC $=12$ como limite minimo de sobrevivência humana. Mais estudos são necessários para se poder determinar a validade da utilização dos limites de corte sugeridos para DEC em países em desenvolvimento.

\section{IMC em Outros Grupos Populacionais}

O IMC também tem sido sugerido como possível indicador do estado nutricional em dois outros grupos populacionais: as gestantes ${ }^{48} \mathrm{e}$ adolescentes. No caso das gestantes, o IMC prégestacional apresenta benefício sobre outros indicadores de massa corporal para estatura, pelo fato dele ser uma razão que não necessita de tabela de referência para se determinar o risco nutricional. Resta validar os limites de corte, sugeridos para tal fim, com outros indicadores tradicionalmente utilizados nesta avaliação, como, por exemplo, o ganho de peso da gestante e o peso da criança ao nascer.

A utilização do IMC na avaliação nutricional de crianças, durante a adolescência, parece pouco apropriada pelo fato do IMC não representar as grandes alteraçס̃es na composição corporal que ocorrem nesta fase da vida. Dados do estudo de saúde e nutrição da população americana (NHANES) $^{32}$ expressam esta idéia (Figura 2). A mediana do IMC permanece comparável entre sexo até aproximadamente os 11 anos de vida, quando os valores das meninas superam os dos meninos, permanecendo superiores até aproximadamente os 17 anos de vida. Já as dobras cutâneas

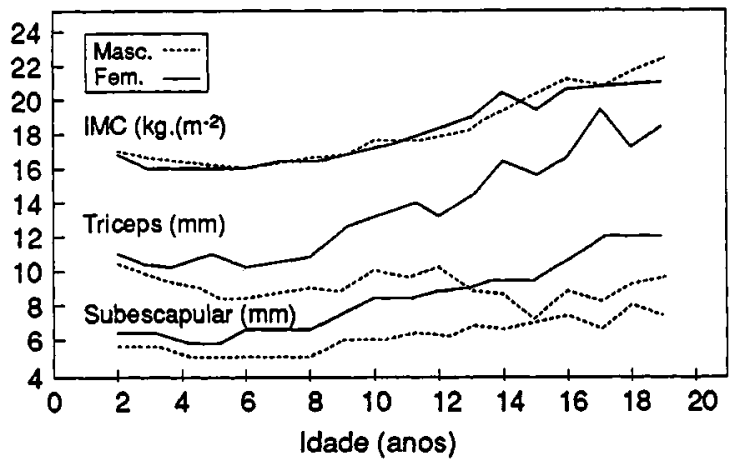

Figura 2. Medianas do índice de massa corporal (IMC) e dobras cutâneas tricipital e subescapular da populaçąo americana em tunção do sexo e idade. Desenhado a partir de dados publicados da NHANES $\| 32$.

tricipital e subescapular evidenciam padröes bem diferenciados entre os sexos, fato bastante marcado para a dobra cutânea tricipital a partir dos 10 anos de idade. Marshall e col..$^{25}$ (1991) compararam a validade de vários indicadores antropométricos de obesidade em função dos valores de composição corporal em crianças de 7 a 14 anos de idade. Como esperado, o somatório de quatro dobras cutâneas mostrou ser mais sensível que o IMC na identificação de obesidade, principalmente em meninas.

\section{IMC e Morbi-mortalidade}

Pelas facilidades de obtenção já discutidas, vários pesquisadores e entidades profissionais têm sugerido o uso do IMC em estudos da relação entre sobrepeso e o risco de mortalidade e morbidade das doenças crônicas $4,16,17,18,33,35,49$. Já que o IMC não mede a composição corporal, ele também não expressa a distribuição da gordura corporal, fator que parece importante na determinação de risco de doenças cardiovasculares, hipertensão, diabetes e acidentes vasculares cerebrais ${ }^{40,43}$. De qualquer forma, a relação entre o risco relativo de mortalidade total e o IMC tem sido identificada como uma curva em "U", assimétrico, sendo que o menor risco (a parte horizontal do $U$ ) fica aproximadamente no intervalo de IMC de 20 a $30 \mathrm{~kg}\left(\mathrm{~m}^{-2}\right)$. Ou seja, os dois extremos de IMC estão associados com maior risco de morbidade e mortalidade $4,32,43,49$. Em estudo da taxa de mortalidade durante 10 anos, de 1,7 milhoes de noruegueses, com idade acima de 15 anos, Waaler ${ }^{43}$ (1988) encontrou diferenças na causa de morte nos dois extremos da distribuição do IMC: as causas de morte associadas com IMC baixo foram tuberculose, câncer pulmonar e doenças pulmonares obstrutivas crônicas, enquanto as causas de mortalidade associadas com IMC alto foram doenças cardio- 


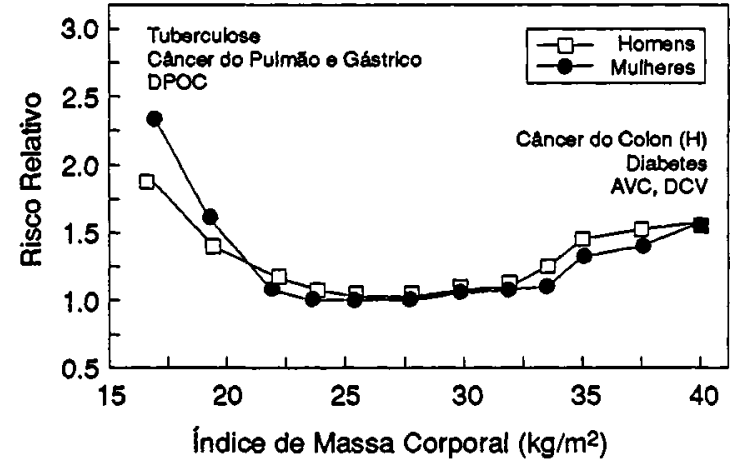

Figura 3. Relaçăo entre o índice de massa corporal (IMC) $\theta$ o risco relativo de mortalidade de 1,7 milhoes de noruegueses durante 10 anos. Desenhando a partir de dados publicados por Waaler43 (1988).

vasculares, diabetes e, para homens, câncer do cólon (Figura 3). O intervalo de 19 a 19,9 do IMC foi o que se associou com a menor mortalidade.

\section{Vigilância Nutricional}

O IMC parece ter bom potencial como indicador do estado nutricional em sistemas de vigilância nutricional, já que os dados de MC e EST são (ou pelo menos deveriam ser) rotineiramente coletados durante consultas de adultos. Uma experiência de vigilância nutricional em unidade básica de serviço, no Rio de Janeiro, mostrou a viabilidade do uso do $\mathrm{IMC}^{1}$. Esta experiência desenvolveu-se num projeto de implantação de um cartão do adulto, onde, além do estado nutricional avaliado pelo IMC, constam também outras observaçōes como controle da pressão arterial, diabetes e nivel de colesterol numa perspectiva de atenção integral ao paciente.

\section{Conclusáo}

Apesar de não indicar a composição corporal, a facilidade de sua mensuração e a grande disponibilidade de dados de massa corporal e estatura, além da sua relação com morbi-mortalidade, parecem ser motivos suficientes para a utilização do IMC como indicador do estado nutricional em estudos epidemiológicos em associação (ou não) a outras medidas antropométricas, até que metodologias de campo, que expressem a composição corporal sejam desenvolvidas para estudos epidemiológicos.

\section{Agradecimentos}

O autor agradece a revisão de uma versão preliminar do manuscrito feita por Denise Oliveira e
Silva, Ricardo Ventura Santos e William Waissmann.

ANJOS, L.A. [Body mass index as a tool in the nutritional assessment of adults: a review]. Rev. Saúde públ., S.Paulo, 26: 431-6, 1992. The limitations and possible uses of the body mass index (BMI) or Quételet index, calculated as the ratio of body mass $(\mathrm{kg})$, and squared stature $(\mathrm{m})$, as an indicator of the nutritional status of adults, are reviewed. The cut-off values for overweight (BMI $\geq 25$ ) and chronic energy deficiency (BMI < 18.5) are discussed in the light of the available international literature. Even though BMI does not assess the body composition (BC) of individuals, the case with which it is measured is a strong argument for the use of BMI in epidemiological studies associated otherwise with other anthropometric measurement until such a time as simpler field methods of the assessment of $\mathrm{BC}$ are developed.

Keywords:Nutritional status. Body mass index. Obesity. Protein-calorie malnutrition.

\section{Referências Bibllográficas}

1. ANJOS, L.A.; SILVA, D.O.; SERRÃO, S.A.; RODRIGUES, C.V.C.S. Vigilância nutricional em adultos: experiência de uma unidade de saúde atendendo popu. lação favelada. Cad. Saúde públ., 8:50-6, 1992.

2. BRAY, G.A. Pathophysiology of obesity. Amer. J. clin. Nutr., 55(Suppl 2): 488-95, 1992.

3. BURSKIRK, E.R. Body composition analysis: the past, present and future. Res. Quart. Exerc. Sport, 58:1-10, 1987.

4. BURTON, B.T.; FOSTER, W.R.; HIRSCH, J.; VANITALLIE, T.B. Health implications of obesity: An NIH consensus development conference. Int. J. Obesity, 9:155$69,1985$.

5. CRONCK, C.E. \& ROCHE, A.F. Race-and sex-specific reference data for triceps and subscapular skinfolds and weight/stature ${ }^{2}$. Amer. J. clin. Nutr., 35:347-54, 1982.

6. DEURENBERG, P.; KOOY, K.; HULSHOF, T.; EVERS, P. Body mass index as a measure of body fatness in the elderly. Eur. J. clin. Nutr., 43:231-6, 1989.

7. FORBES, G. B. The adult decline in lean body mass. Human Biol., 48:161-73, 1976.

8. FRISANCHO, A.R. \& FLEGEL, P. N. Relative merits of old and new indices of body mass with reference to skinfold thickness. Amer. J. clin. Nutr., 36:697-9, 1982.

9. GARN, S. M.; LEONARD, W. R.; HAWTHORNE, V. M. Three limitations of the body mass index. Amer.J. clin. Nutr., 44:996-7, 1986.

10. GARROW, J. S. Treat obesity seriously: a clinical manual. London; Churchill Livingstone, 1981.

11. GARROW, J. S. Indices of adiposity. Nutr. Abstr. Rev. Ser. A., 53:697-708, 1983.

12. GARROW, J. S. Three limitations of the body mass index. Amer. J. clin. Nutr., 47:553, 1988.

13. GARROW, J. S. \& WEBSTER, J. Quetelet's index $\left(W / \mathrm{H}^{2}\right)$; as a measure of fatness. Int. J. Obesity, 9:147-53, 1985.

14. GUEDES, D. P. Estudo da gordura corporal através da mensuração dos valores de densidade corporal e da espessura de dobras cutâneas em universitários. Santa Maria, 1985. [Dissertação de Mestrado - Universidade Federal de Santa Maria]. 
15. HENRY, C. J. K. Body mass index and the limits of human survival. Eur. J. clin. Nutr., 44:329-35, 1990.

16. HIGGINS, M.; KANNEL, W.; GARRISON, R.; PINSKY, J.; STOKES, J. Hazards of obesity-the Framingham experience. Acta med. scand. Suppl., (723):23-36, 1988.

17. HOFFMANS, M. D. A.; KROMHOUT, D.; LEZENNECOULANDER, C. The impact of body mass index of 78612 18-year old Dutch men on 32-year-mortality from all causes. J. clin. Epidem., 41:749-56, 1988.

18. INSTITUTO NACIONAL DE ALIMENTAÇĀO E NUTRIÇÃO. Pesquisa nacional de saúde e nutrição: perfil de crescimento da populaçâo brasileira de 0 a 25 anos. Brasilia, 1990.

19. JAMES, W.P.T. \& SCHOFIELD, E.C. Human energy requirements: a manual for planners and nutritionists. Oxford, Oxford University Press, 1990.

20. JAMES, W.P.T;; FERRO-LUZZI, A.; WATERLOW, J.C. Definition of chronic energy deficiency in adults. Report of a Working Party of the International Dietary Energy Consultative Group. Eur. J. Clin. Nutr ., 42:969-81, 1998.

21. KEYS, A.; FIDANZA, F.; KARVONEN, M.J.; KIMURA, N.; TAYLOR, H.L. Indices of relative weight and obesity. J. chron. Dis., 25:329-43, 1972.

22. LEE, J.; KOLONEL, L.N.; WARD HINDS, M. Relative merits of the weight-corrected-for-height indices. Amer. J. clin. Nutr., 34:2521-9, 1981.

23. LOLIO, C.A. \& LATORRE, M.R.D.O. Prevalência de obesidade em localidade de Estado de São Paulo, Brasil, 1987. Rev. Saúde públ., S.Paulo, 25:33-6, 1991.

24. LUKASKI, H.C. Methods for the assessment of human body composition: traditional and new. Amer. J. clin. Nutr., 46:537-56, 1987.

25. MARSHALL, J.D.; HAZLETT, C.B.; SPADY, D.W.; CONGER, P.R.; QUINNEY, H.A. Validity of convenient indicators of obesity. Human Biol ., 63:137-53,1991.

26. MARTORELL, R.; MENDONZA, F.; CASTILLO, R. Poverty and stature in children. In: Waterlow, J.C., ed. Linear growth retardation in less developed countries. New York, Raven Press, 1988. p. 57-73. (Nestle Nutr. Workshop Series, 14).

27. MCLAREN, O.S. Three limitations of body mass index. Amer. J. clin. Nutr., 46:121, 1987.

28. MICOZZI, M.S.; ALBANES, D.; JONES, D.Y.; CHUMLEA, W.C. Correlations of body mass indices with weight, stature, and body composition in men and women in NHANES I and II. Amer. J. clin. Nutr., 44:725-31, 1986.

29. MUST, A.; DALLAL, G.E.; DIETZ, W.H. Reference data for obesity: 85 th and 95 th percentiles of body mass in$\operatorname{dex}(W T / H T 2)$ : and triceps skinfold thickness. Amer, $J$. clin. Nutr., 53:839-46, 1991.

30. MUST, A.; DALLAL, G.E.; DIETZ, W.H. Reference data for obesity: 85 th and 95 th percentiles of body mass in$\operatorname{dex}(\mathrm{WT} / \mathrm{HT} 2)$ : a correction. Amer. J. clin. Nur., 54:773, 1991.

31. NATIONAL ACADEMY OF SCIENCES. Diet and health: scientific concepts and principles. Nutr. Rev., 45:276-87, 1987.

32. NATTONAL CENTER FOR HEALTH STATISTICS. Anthropometric reference data and prevalence of overweight, United States, 1976-80. Vital and Health Statistics. Hyattsville, MD. DHHS Pub. no. (PHS) 87-1688, 1987.
33. NATTONAL INSTITUTES OF HEALTH CONSENSUS DEVELOPMENT CONFERENCE STATEMENT. Health implications of obesity. Ann. intern. Med., 103 (6, pt.2):1073-7, 1985.

34. NORGAN, N.G. Body mass index and body energy stores in developing countries. Eur. J. clin. Nutr., 44 (suppl. 1):79-84, 1990.

35. PI-SUNYER, F.X. Health implications of obesity. Amer.J. clin. Nutr., 53:1595S-603S, 1991.

36. QUETELET, A. Antropométrie ou mesure des différentes facultés de l'homme. Bruxelles, C. Muquardt, 1870.

37. ROCHE, A.F. Anthropometric methods: new and old, what they tell us. Int.J. Obesity, 8:509-23, 1984.

38. ROLLAND-CACHERA, M.F.; COLE, T.J.; SEMPE, M.; TICHET, J.; ROSSIGNOL, C.; CHARRAUD, A. Body mass index variations: centiles from birth to 87 years. Eur. J. clin. Nutr., 45:13-21, 1991.

39. ROOKUS, M.A.; BUREMA, J.; HOF, M.A.V.; DEURENBERG, P.; WIEL-WETZELS, W.A.M.V.; HAUTVAST, J.A.G.J. The development of the body mass index in young adults. I: Age-reference curves based on a four-year mixed-longitudinal study. Human Biol., 59:599-615, 1987.

40. SEIDELL, J.C.; BAKX, J.C.; DE BOER, E.; DEURENBERG, P.; HAUTVAST, J.A.G.J. Fat distribuition of overweight persons in relation to morbidity and subjective health. Int. J. Obesity, 9:363-74, 1985 .

41. SMALLEY, K.J.; KNERR, A.N.; KENDRICK, Z.V.; COLLIVER, J.A.; OWEN, O.E. Reassessment of body mass indices. Amer. J. clin. Nutr., 52:405-8, 1990.

42. VANITALLIE, T.B.; YANG, M.; HEYMSFIELD, S.B.; FUNK, R.C.; BOILEAU, R.A.R.A. Height-nomalized indices of the body's fat-free mass and fat mass: potentially useful indicators of nutritional status. Amer. J. clin. Nutr., 52:953-9, 1990.

43. WAALER, H.T. Hazard of obesity: the Norweigian experience. Acta med. scand. Suppl., (723):17-21, 1988.

44. WOMERSLEY, J. \& DURNIN, J.V.G.A. A comparison of the skinfold method with extent of "overweight" and various weight-height relationships in the assessment of obesity. Brit. J. Nutr., 38:271-82, 1977.

45. WORLD HEALTH ORGANIZATION. Energy and protein requirements. Geneva, 1985. (Technical Report Series 724).

46. WORLD HEALTH ORGANIZATION. Use and interpretation of anthropometric indicators of nutritional status. Bull. wld. Hllh. Org., 64:929-41, 1986.

47. WORLD HEALTH ORGANIZATION. Diet nutrition and the prevention of chronic diseases. Geneva, 1990. (Technical Report Series 797).

48. WORLD HEALTH ORGANIZATION. Matemal anthropometry for prediction of pregnancy outcomes; memorandum from a USAID/WHO/PAHO/Mother Care Meeting. Bull. wld. Hlh. Org., 69:523-32, 1991.

49. WORLD HYPERTENSION LEAGUE. Weight control in the management of hypertension. Bull. wld Hlth Org, 67:245-52, 1989.

Recebido para publicaçâo em 6.5.1992. Reapresentado em 14.8.1992. Aprovado para publicação em 1.9.1992. 